Review Article: Open Access

\title{
Levothyroxine for Hypothyroidism: Current Status of Biowaiver and Bioequivalence Studies
}

\author{
Núñez-Guzmán NA ${ }^{1 *}$, Muñoz-Ibarra Al', Ruiz-Molina $D^{1,2}$, Figueroa-Núñez $B^{1}$ and García-Pérez $M E^{3}$ \\ ${ }^{1}$ Chronic Disease and Special Procedures Clinic, CECYPE, México \\ ${ }^{2}$ Center for Research and Assistance in Technology and Design of the State of Jalisco, A.C. (CECYPE), México \\ ${ }^{3}$ Faculty of Chemistry Pharmacology, Michoacan University of San Nicolás de Hidalgo, UMSNH, México
}

*Corresponding author: Núñez-Guzmán NA, Chronic Disease and Special Procedures Clinic, Michoacan University of San Nicolás de Hidalgo, Fray Bernardino de Sahagún \#101, Morelia, Michoacán, México, Tel: 01-443-3-23-2828, Fax: 3-23-2900, E-mail: nora.nunez@cecype.com

\begin{abstract}
Hypothyroidism is a common disease that needs to be closely treated, in order to reduce adverse symptoms and pathophysiological effects. For thyroid hormone replacement is preferred a therapy with a synthetic form of thyroxine (levothyroxine), a drug recognized by its Narrow Therapeutic Index. Generic levothyroxine can be an economic alternative for Hypothyroidism treatment. However, bioequivalence studies with levothyroxine are considered controversial. The aim of this review is to discuss aspects related to bioequivalence and biowaiver studies for levothyroxine considering the point of view of various regulatory agencies worldwide. Also, alternative strategies are proposed to perform bioequivalence with this drug
\end{abstract}

\section{Keywords}

Levothyroxine bioequivalence, Study design, Narrow Therapeutic Index (NTI), Biowaiver

\section{Introduction}

It is currently known that hypothyroidism is a relatively common disease, in which early diagnosis helps to reduce mortality by circulatory and cardiovascular disorders preventing progression to myxedema coma, which has high mortality. Hypothyroidism is a disease with an incidence of 3.5 per 1000 women and 0.6 per 1000 men and increases with age up to 14 per 1000 women between 75 and 80 -years-old. A $10 \%$ is registered in the general population over 60 years. When making a diagnosis of primary hypothyroidism the treatment is initiated with thyroid hormone (levothyroxine) [1]. Subclinical hypothyroidism and subclinical hyperthyroidism are associated with adverse symptoms and pathophysiological effects.

Levothyroxine is one of the most commonly prescribed medications, with a Narrow Therapeutic Index, only for Food and Drug Administration (FDA) [2]. The consequence of failed dosage or a bad follow-up produce cardiovascular problems such as hypercholesterolemia, increased fibrinolytic activity, systolic and diastolic dysfunction, atherosclerosis, and myocardial infarction. On the other hand, mild thyrotoxicosis increases the likelihood of arrhythmias, ventricular dysfunction, and reduced exercise performance leading to a possible cardiovascular death. In the elderly, iatrogenic hyperthyroidism has been associated with the development of osteoporosis and approximately 600,000 elderly patients may be at risk for bone demineralization because levothyroxine overdose [3]. Consequently, to reduce this illness it is very important to have a close clinical follow-up of patients.

The first synthetic levothyroxine was introduced in 1958 [4]. Its clinical effectiveness was well established through years of clinical use. Nevertheless there is a high degree of uncertainty about the use of this medicament, which has been due to: a) product stability (i.e., shelflife); b) formulation consistency over time within a given "brand"; c) bioequivalence studies between brands; d) levothyroxine degrades quickly with exposure to light, moisture, oxygen, and carbohydrate excipients; e) significant changes in formulation occurring over time as firms attempted to improve product stability [5].

Early diagnosis and treatment of hypothyroidism restores the physical and cognitive function of patients, with a better willingness to perform daily activities [1]. Treatment is generally lifelong [6]. The cost generated for the patient and public health system, is very high and sometimes can become the cause of improper handling, with all the complications that this entails. So, it is important to have generic drugs available in the market, which may be offered at lower costs, improving the quality of life of patients. It is a priority topic, but such generic drugs must first prove to be safe and effective during pharmacokinetic studies. Biowaiver can become an important and cost-saving tool for the development and approval of generic drugs. The aim of this review is to discuss aspects related to bioequivalence and biowaiver studies for levothyroxine considering the point of view of various regulatory agencies worldwide. Also, alternative strategies are proposed to perform bioequivalence with this drug.

\section{Historical Background of Levothyroxine}

In 1997, FDA detected stability and potency problems related to levothyroxine and declaring orally administered levothyroxine sodium drug products to be new drugs and requiring new drug application (NDA) submissions [7].

Citation: Núñez-Guzmán NA, Muñoz-Ibarra Al, Ruiz-Molina D, Figueroa-Núñez B, GarcíaPérez ME (2017) Levothyroxine for Hypothyroidism: Current Status of Biowaiver and Bioequivalence Studies. Int Arch Clin Pharmacol 3:009

International Library Copyright: @ 2017 Núñez-Guzmán NA, et al. This is an open-access article distributed under the terms of the Creative Commons Attribution License, which permits unrestricted use, distribution, and reproduction in any medium, provided the original author and source are credited. 
Prior to August 2000, levothyroxine sodium was an unapproved marketed drug. Case reports in the literature suggested that therapeutic failures had occurred when patients received a refill of the same product for which they had been previously stable. Of the 58 case reports of therapeutic failure received by the FDA from 19871994 , nearly half occurred when patients received a refill of a product on which they had been stable for years [5].

With regard to assure the appropriate bioequivalence conditions, FDA on December 2000, published the "Guidance for Industry: Levothyroxine Sodium Tablets-In Vivo Pharmacokinetic and Bioavailability Studies and In Vitro Dissolution Testing" and proposed to determine biodisponibility of levothyroxine sodium drug products. In 2006, FDA requested product stability data from levothyroxine sodium drug manufacturers, which was then provided to the agency in the spring of that year [7].

In 2012 the health authorities in Mexico (Federal Commission for Sanitary Risk Protection - COFEPRIS), stated that bioequivalence studies with levothyroxine were not possible, every levothyroxine product must be submitted as a new product and demonstrate their quality, safety and efficacy on individual basis. In this way levothyroxine was not interchangeable, so it should not be replaced in therapeutic practice. Because this synthetic hormone is used as replacement for thyroid function, it is considered a drug risk. In the case of medicines already registered, it was recommended that studies were undertaken in Phase IV by following a permanent pharmacovigilance program [8].

In 2014 FDA published the "Draft Guidance on Levothyroxine Sodium" where they recommended a bioequivalence study in fasted conditions, single dose, four-way, fully replicated without stability or power requirements [9].

\section{Levothyroxine as a Narrow Therapeutic Index (NTI)}

FDA defines NTI drugs as those which have less than a 2 -fold difference in median lethal dose (LD50) and median effective dose (ED50) values, or have less than a 2 -fold difference in the minimum toxic concentrations and minimum effective concentrations in blood. Therefore, safe and effective use of these drugs requires careful dosage titration and patient monitoring [10]. FDA has concluded that levothyroxine sodium is a NTI drug based on [9]: a) the range between serum levothyroxine therapeutic and toxic concentrations is narrow; b) some levothyroxine-associated toxicities are serious and/or irreversible; c) sub-therapeutic levothyroxine concentrations result in inadequate treatment and lead to poor clinical outcomes; d) levothyroxine sodium requires individual dose titration to achieve a satisfactory balance between maximizing efficacy and minimizing serious dose-related toxicity; e) therapeutic drug monitoring based on serum Thyroid Stimulating Hormone and total or free-levothyroxine levels is routinely employed to facilitate levothyroxine dose titration; and $\mathrm{f}$ ) levothyroxine has small-to-medium intra subject variability.

LX Yu, et al. defined NTI drugs as those drugs where small differences in dose or blood concentration may lead to serious therapeutic failures and/or adverse drug reactions that are lifethreatening or result in persistent or significant disability or incapacity [11].

According to the European Medicine Agency (EMA) in specific cases of products with a NTI, the acceptance confidence interval for a bioequivalence study may need to be tightened. For EMA it is not possible to define a set of criteria to categorize drugs as either NTI drugs or not and a judgement must be made in each individual case. Likewise, the need for narrowing the acceptance interval for both area under the curve (AUC) and peak concentration $\left(\mathrm{C}_{\max }\right)$ or for AUC only (to $90-111.11 \%$ ) should be determined on a case by case basis [12].

Health Canada Agency defined the NTI ("critical dose drugs") as those drugs where comparatively small differences in dose or concentration lead to dose- and concentration-dependent, serious therapeutic failures and/or serious adverse drug reactions [13]. For these drugs: a) the $90 \%$ confidence interval of the relative mean AUC of the test to reference formulation should be within $90 \%$ to $112 \%$ inclusive and; b) the $90 \%$ confidence interval of the relative mean $\mathrm{C}_{\text {max }}$ of the test to reference formulation should be between $80 \%$ and $125 \%$ inclusive [13].

According to the Guidelines for the evaluation of interchangeability medicines containing NTI drugs and critical dose, COFEPRIS, defines a NTI drug when in preclinical studies, the relationship is less than 2 between lethal dose $50\left(\mathrm{LD}_{50}\right)$ and the effective dose $50\left(\mathrm{ED}_{50}\right)$. Due in clinical trials cannot be using these parameters, it is recommended to use the ratio between the toxic concentration 10 and the effective concentration 90 [14]. The acceptance interval for AUC should be tightened from the conventional $80-125 \%$ to $90-111.11 \%$. $\mathrm{C}_{\max }$ interval must be included between 80 and 125\% [14].

\section{Biowaiver}

Health Canada Agency under Division 8 of the Food and Drug Regulation, inform on May 30, 2014 the guidance document: Biopharmaceutics Classification System applicable only for class I and III drugs [15]. This guideline does not mention anything about levothyroxine.

However, Food and Drug Administration, in Guidance for Industry has a specific guideline "Levothyroxine Sodium Tablets - In Vivo Pharmacokinetic and bioavailability Studies and In Vitro Dissolution Testing". In this guidance, FDA states that biowaiver for tablets strengths is possible only if: a) three in vivo studies, low, middle and high strength are bioequivalent; $b$ ) tablets evaluated only differ in the amount of levothyroxine sodium and filler needed to maintain the tablet weights; and c) dissolution profile are similar across tablets strengths using a f2 test [16].

On December 2014, FDA published a "Draft guidance on Levothyroxine Sodium" and recommended that the waiver for in vivo testing is possible for $0.025,0.05,0.075,0.088,0.1,0.112,0.123,0.137$, $0.15,0.175$ y $0.2 \mathrm{mg}$ with an acceptable bioequivalence study on the $0.3 \mathrm{mg}$ strength, acceptable in vitro dissolution testing of all strength and proportional similarity of the formulations across all strengths [9].

Medicines and Healthcare Products Regulatory Agency on January 2013 in "Levothyroxine Tablet Products: A Review of Clinical \& Quality Considerations" says that levothyroxine sodium has atypical solution properties coupled with an extremely slow intrinsic dissolution rate. As a result, a BCS-based biowaiver for bioequivalence to reference products is not accepted for any new application for a marketing authorization [17].

World Health Organization states the biowaivers are applicable for immediate-release solid oral dosage formulations containing one or more drugs, however, it only apply for a list of ten drugs published in a guidance document, levothyroxine is not mentioned, even there is not recommendations about bioequivalence studies [18].

EMA in the guideline of the investigation of bioequivalence states that biowaiver is applicable for additional strength when the composition of strengths are quantitatively proportional. However, if the amount of the active substance is less than $5 \%$ of the tablet core weight, the amounts of the different core excipients or capsule content are the same for the concerned strengths and only the amount of active substance is changed. Other requirement is the linear pharmacokinetic. Indeed, the bioequivalence study should be conducted at the highest strength, another strength is acceptable for safety/tolerability reasons. When the drug does not follow a linear pharmacokinetics the bioequivalence evaluation should be conducted with the highest and lowest strength or the strength in the linear range, so, two bioequivalence studies are needed. When bioequivalence assessment at more than two strengths is needed (for deviation form proportional composition), a bracketing approach may be used, if the strengths selected represent the extremes (the highest and the lowest, or the strengths differing most in composition), so any differences in composition in the remaining strengths is covered by two conducted studies [19]. 
In Mexico, COFEPRIS in 2012 indicated that levothyroxine was not interchangeable [8]. On October 2016, this agency states that levothyroxine allows the bioequivalence trials, also the biowaiver is possible for all the dosage performing a bioequivalence study with the highest strength [20].

\section{Levothyroxine Bioequivalence Studies}

In most cases, the recommended bioequivalence study design for any drug is a randomized, single-dose, two-way crossover in healthy normal subjects. Patients should be used for reasons of safety only. Japan recommends using achlorhydric subjects in bioequivalence studies. Most of the jurisdictions and organizations, including Mexican normativity, recommend that a bioequivalence study enrolls at least 12 subjects; China suggest 18-24 and Japan the sufficient number to shown bioequivalence. Regarding the eligibility of subjects, all agencies recommend age range and body weight restrictions. Most guidance's recommend that both gender subjects be enrolled [21].

Today there is no global agreement on what kind of specific clinical trials are the best to demonstrate the interchangeability of levothyroxine.

The Medicines and Healthcare products Regulatory Agency (MHRA) as well FDA recommend that major formulation and manufacturing changes on products that containing levothyroxine, should be supported by bioequivalence. However, establishing bioequivalence and its correlation to therapeutic equivalence for endogenous substances in general, and for levothyroxine in particular, continues to be a challenge [17].

There are some articles on levothyroxine bioequivalence studies which have demonstrated the interchangeability of test drugs with respect to the reference products. Di Girolamo, et al. described the bioequivalence of two levothyroxine tablet formulations with and without mathematical adjustment for basal thyroxine levels in healthy Argentinian volunteers. In this work the bioequivalence between the products was demonstrated using a single-dose, randomized, openlabel, crossover study [22]. Koytchev and Lauschner published an article entitled "Bioequivalence Study of Levothyroxine Tablets Compared to Reference Tablets and an Oral Solution" in which the bioequivalence could also be demonstrated [23].

However, the determination of bioequivalence for thyroid products is still controversial. The controversy is based on several issues, some of these are: a) the interference of the accurate analysis of plasma levels of levothyroxine following oral administration with respect to endogenous levels (which are themselves variable); b) the designation of levothyroxine as an NTI drug; c) the relevance of levothyroxine compared with TSH as the bioequivalence "indicator"; d) the need for use of a high dose of levothyroxine in bioequivalence studies and e) the potential for confounding carryover effects [24].

On 2000, FDA accepted the bioequivalence study with a singledose, two-treatment, two-sequence crossover design with a washout period between treatments of at least 35 days [16]. More recently on December 2014, this agency changed the recommendations and published a new guide which indicates a single-dose, four-way, fully replicated crossover in vivo, in fasting conditions and because of the long elimination half-life, hence adequate washout periods should be ensured between treatments in the crossover study [9].

FDA proposes that the bioequivalence of NTI drugs can be determined using a scaling approach with a four-way, fully replicated, crossover design study in healthy subjects in order to allow the simultaneous equivalence comparison of the mean and intra subject variability of the test and reference products. The proposed bioequivalence limits for NTI drugs of $90 \%-111.11 \%$ would be scaled based on the intra subject variability of the reference product. The proposed study design and data analysis should provide greater assurance of therapeutic equivalence of NTI drug products [11].

The European Medicines Agency (EMA) does not have a specific guideline for levothyroxine. In Brazil, there are no guidelines on interchangeability in relation to thyroid hormones [25]. Recently in Mexico, on October $07^{\text {th }} 2016$, it has been authorized bioequivalence testing for levothyroxine by the "General Council of Health", but there is still no position on the study design, which dose has to be used or if it is necessary to consider this drug as an NTI drug. This statement only mentions that "special procedures must be performed" without specifying which procedures relate [20]. No official communicates indicates that the $2 \times 2$ crossover design could be the study recommended.

\section{Conclusions}

The FDA guidance on bioequivalence studies using levothyroxine advises that a single dose of $0.6 \mathrm{mg}$ is administered to healthy volunteers and this dose also appears to be generally well tolerated. Therefore, acute toxicity with well over double the daily requirement of levothyroxine does not seem to pose safety risks, at least over the short term [17]. FDA considers levothyroxine as a NTI drug and recommends performing a study with four periods (four drug administration by each volunteer). However, FDA does not specify the tighten of the confidence intervals as EMA guideline. The Biowaiver, could be possible for levothyroxine according to the Biopharmaceutics Classification System, as a class I drug (high solubility, high permeability [26] and considering agencies recommendations above mentioned).

Taking into account that there is a lack of consensus regarding the design for levothyroxine bioequivalence studies we propose the following strategies: 1 ) a crossover $2 \times 2$ design instead of fourway, fully replicated to reduce the intake of the drug by healthy volunteers and the possibility of side effects, decreasing the time of the entire study (washout period should be at least 30 days) and also due the low intra subject variability of this drug; 2 ) the "Bracketing approach", could be the best method for biowaver in which two bioequivalence studies should be performed selecting the extremes in the strengths (e.g. the highest and the lowest) in fasting and fed conditions, according with EMA's guidelines [12]; 3) it is important to consider levothyroxine as a NTI drug, because small changes in the doses lead major physiological effects in patients including serious adverse reactions; 4 ) the bioequivalence confidence interval should be reduced to $90-111.11 \%$ for AUC according to the international guidelines recommendations for NTI drugs; 5) to avoid the loss of potency of levothyroxine we recommend to perform the in vitro stability test more strictly (temperature, humidity, light) reducing the variability in the acceptance for this test.

Overall, there is currently an agreement between the regulatory agencies worldwide on the possibility of bioequivalence for levothyroxine products. Nevertheless, there is still no agreement on the design of such studies. Moreover, whether levothyroxine should be considered as NTI drug is also a matter of debate. So, each country must follow its own laws while global consensus is achieved. However, while agencies decide, patients are waiting to obtain a generic product.

\section{References}

1. Medráno Ortíz-de Zárate ME, Santillana-Hernández SP. Torres-Arreola LP Gómez-Díaz RA, Rivera-Moscoso R, et al. (2012) Guía de Práctica Clínica Diagnóstico y Tratamiento del hipotiroidismo primario en adultos. Rev Med Inst Mex Seguro Soc 50: 71-80.

2. Wartofsky $L$ (2002) Levothyroxine: therapeutic use and regulatory issues related to bioequivalence. Expert Opin Pharmacother 3: 727-732.

3. Blakesley V, Awni W, Locke C, Ludden T, Granneman GR, et al. (2004) Are Bioequivalence Studies of Levothyroxine Sodium Formulations in Euthyroid Volunteers Reliable? Thyroid 14: 191-200.

4. Santella TM, Wertheimer Al (2005) The levothyroxine spectrum: Bioequivalence and cost considerations. Formulary Journal Pharmacy.

5. JohnsonSB(2003)Endogenous SubstanceBioavailability and Bioequivalence: Levothyroxine Sodium Tablets; Division of Pharmaceutical Evaluation II FDA / CDER / OPS / OCPB.

6. Gómez-Meléndez GA, Ruiz-Betanzos R, Sánchez-Pedraza V, SegoviaPalomo A, Mendoza-Hernández CF, et al. (2010) Hipotiroidismo Med Int Mex 26: $462-471$. 
7. FDA (2007) Questions and Answers on Levothyroxine Sodium Products.

8. (2012) Official statement about bioequivalence studies for levothyroxine issued by Federal Commission for Sanitary Risks Protection in Mexico (COFEPRIS).

9. FDA (2014) Draft Guidance on Levothyroxine Sodium.

10. FDA (2016) Code for Federal Regulations Title 21.

11. Yu LX, Jiang W, Zhang X, Lionberger R, Makhlouf F, et al. (2015) Nove bioequivalence approach for narrow therapeutic index drugs. Clin Pharmaco Ther 97: 286-291.

12. EMA (2008) Guideline on the investigation of bioequivalence.

13. (2012) Guidance Document Comparative Bioavailability Standards: Formulations Used for Systemic Effects. Health Products and Food Branch.

14. Guide for the evaluation of interchangeability of medicinal products containing narrow therapeutic drugs and critical dose, COFEPRIS.

15. HPFB (2016) Health Product and Food Branch. Obtained from Health Canada.

16. FDA (2000) Guidance for Industry.

17. MHRA (2013) Medicines and Healthcare products Regulatory Agency.
18. WHO (2011) WHO Prequalification of Medicines Programme.

19. EMA (2010) Committe for Medical Products for Human Use (CHMP).

20. General Health Council (2016) Agreement that determines the type of test to demonstrate interchangeability of generic drugs. Official Diary.

21. Davit B, Braddy AC, Conner DP, Yu LX (2013) International Guidelines for Bioequivalence of Systemically Available Orally Administered Generic Drug Products: A Survey of Similarities and Differences. AAPS J 15: 974-990.

22. Di Girolamo G, Keller GA, De los Santos AR, Schere D, Gonzalez CD (2008) Bioequivalence of Two Levothyroxine Tablet Formulations Without and With Mathematical Adjustment for Basal Thyroxine Levels in Healthy Argentinian Volunteers: A Single-Dose, Randomized, Open-Label, Crossover Study. Clinical Thera 30: 2015-2023.

23. Koytchev R, Lauschner R (2004) Bioequivalence study of levothyroxine tablets compared to reference tablets and an oral solution. Arzneimittelforschung 54 : 680-684.

24. Bolton S (2005) Bioequivalence Studies for Levothyroxine. AAPS J 7: E47-53.

25. Ward LS (2011) Levothyroxine and the problem of interchangeability of drugs with narrow therapeutic index. Arq Bras Endocrinol Metabol 55: 429-434.

26. BCS Classification (2016) tsrlinc. The Drug Delivery Foundation. 